\title{
A text mining approach for the extraction of kinetic information from literature
}

\author{
Ana Alão Freitas 1, Hugo Costa 2, Miguel Rocha 1, and Isabel Rocha 1 * \\ 1 Centre Biological Engineering, School of Engineering, University of \\ Minho, Braga, \\ Portugal \\ 2 SilicoLife Lda, Rua do Canastreiro, 15, 4715-387 Braga, Portugal \\ *irocha@deb.uminho.pt
}

Keywords: Enzyme kinetics, metabolic models, text mining, name entity recognition, relation extraction, databases.

Systems biology has fostered interest in the use of kinetic models to better understand the dynamic behavior of metabolic networks in a wide variety of conditions. Unfortunately, in most cases, data available in different databases are not sufficient for the development of such models, since a significant part of the relevant information is still scattered in the literature. Thus, it becomes essential to develop specific and powerful text mining tools towards this aim.

In this context, this work has as main objective the development of a text mining tool to extract, from scientific literature, kinetic parameters, their respective values and their relations with enzymes and metabolites. The pipeline proposed integrates the development of a novel plug-in over the text mining tool @Note2. Overall, the results validate the developed approach. 